\title{
On the N-Wave Equations and Soliton Interactions in Two and Three Dimensions
}

Vladimir S. Gerdjikov

Bulgarian Academy of Sciences,, gerjikov@inrne.bas.bg

Rossen Ivanov

Technological University Dublin, rossen.ivanov@tudublin.ie

Assen V. Kyuldjiev

Bulgarian Academy of Sciences, kyuljiev@inrne.bas.bg

Follow this and additional works at: https://arrow.tudublin.ie/scschmatart

Part of the Mathematics Commons, Non-linear Dynamics Commons, and the Partial Differential Equations Commons

\section{Recommended Citation}

V. Gerdjikov, R. Ivanov and A. Kyuldjiev, On the N-wave equations and soliton interactions in two and three dimensions, Wave Motion, 48 (2011) 791-804. Available from the publisher here http://www.sciencedirect.com/science/journal/01652125/48 doi: 10.21427/pbt8-5p79

This Article is brought to you for free and open access by the School of Mathematics at ARROW@TU Dublin. It has been accepted for inclusion in Articles by an authorized administrator of ARROW@TU Dublin. For more information, please contact arrow.admin@tudublin.ie, aisling.coyne@tudublin.ie,gerard.connolly@tudublin.ie. Funder: Science Foundation Ireland(Grant No. 09/RFP/ MTH2144) 


\title{
On the $N$-wave equations and soliton interactions in two and three dimensions
}

\author{
V. S. Gerdjikov ${ }^{1}$, R. I. Ivanov ${ }^{2}$ and A. V. Kyuldjiev ${ }^{1}$ \\ ${ }^{1}$ Institute for Nuclear Research and Nuclear Energy \\ Bulgarian academy of sciences, 1784 Sofia, Bulgaria \\ ${ }^{2}$ School of Mathematical Sciences, Dublin Institute of Technology, \\ Kevin Street, Dublin 8, IRELAND
}

April 20, 2011

\begin{abstract}
Several important examples of the $N$-wave equations are studied. These integrable equations can be linearized by formulation of the inverse scattering as a local Riemann-Hilbert problem (RHP). Several nontrivial reductions are presented. Such reductions can be applied to the generic $N$ wave equations but mainly the 3 - and 4 -wave interactions are presented as examples. Their one and two-soliton solutions are derived and their soliton interactions are analyzed. It is shown that additional reductions may lead to new types of soliton solutions. In particular the 4 -wave equations with $\mathbb{Z}_{2} \times \mathbb{Z}_{2}$ reduction group allows breather-like solitons. Finally it is demonstrated that RHP with sewing function depending on three variables $t, x$ and $y$ provides some special solutions of the $N$-wave equations in three dimensions.
\end{abstract}

\section{Introduction}

The theory of solitons and its applications to wave-wave interactions in nonlinear media is by now a well established topic in today mathematical physics.

It attracted scientists from several different areas of mathematics: spectral theory, dynamical systems, Lie algebras, Hamiltonian dynamics, differential geometry, see $[23,20,26,27,1,4,12,3,24]$ and the numerous references therein. These equations attracted also the attention of a number of physicists because they find important applications in fluid mechanics, nonlinear optics, superconductivity, plasma physics etc. As a result many different approaches for investigating the soliton equations and constructing their Lax representations, soliton solutions, integrals of motion, Hamiltonian hierarchies etc. were developed, see $[28,20,2,23,15,19,18,27,1]$. Of course, it is not possible in a short paper to list all important references that cover the broad topics mentioned above. 
The inverse scattering method has been applied to many physically important multidimensional evolution equations including the $N$-wave equation, Davey-Stewartson, Kadomtsev-Petviashvilli etc. [25, 15, 16, 17, 26, 27, 28, 18, $19,5,22]$. They have been treated by nonlocal generalizations of the RiemannHilbert problem and by the $\bar{\partial}$-method.

Unlike the usual approach, starting with the Lax pair formulation, our starting point is the RHP formulation of the scattering problem. It is an effective tool for constructing the soliton solutions and analysis of their properties. We will show also that the RHP gives clearer prospects for multidimensional generalizations of the Inverse Scattering Method.

Our aim in this paper is to focus on the different types of soliton solutions of $N$-wave equations illustrating them by nontrivial examples for $N=3$ and $N=4$. At first we do so for the standard soliton theory devised to solve soliton equations in two-dimensional space-time. Along with the generic soliton solutions in Section 3 we derive doublet and quadruplet solitons for the 4 -wave equations. Studying their asymptotics for $x$ and $t$ tending to $\pm \infty$ allows one to describe their interactions. In Section 4 we derive the 2 -soliton solution of the 3 -wave equations. In the last Section 5 we show how these results can be generalized to 3 -dimensional space-time. We end with brief discussion and conclusions.

\section{Preliminaries}

\subsection{The RHP and the soliton solutions}

We will start with a matrix-valued RHP

$$
\begin{aligned}
\xi^{+}(x, t, \lambda) & =\xi^{-}(x, t, \lambda) G(x, t, \lambda), \quad \lambda \in \mathbb{R} \\
\lim _{\lambda \rightarrow \infty} \xi^{ \pm}(x, t, \lambda) & =\mathbb{1} .
\end{aligned}
$$

The second line in (2.1) is known as the canonical normalization condition. Roughly speaking the RHP consists in the following: Given the sewing function $G(x, t, \lambda)$ on the real axis reconstruct the two functions $\xi^{+}(x, t, \lambda)$ and $\xi^{-}(x, t, \lambda)$ analytic for $\lambda \in \mathbb{C}_{+}$and $\lambda \in \mathbb{C}_{-}$respectively and satisfying the normalization condition.

We also assume that the sewing function $G(x, t, \lambda)$ depends on two auxiliary parameters: $t$ and $x$, as follows:

$$
i \frac{\partial G}{\partial x}-\lambda[J, G(\vec{x}, t, \lambda)]=0, \quad i \frac{\partial G}{\partial t}-[f(\lambda) I, G(\vec{x}, t, \lambda)]=0,
$$

where $J$ and $I$ are constant elements taking values in $\mathfrak{h}$ - the Cartan subalgebra of $\mathfrak{g}$.

The list of integrable NLEE in 2-dimensional space-time starts with the paradigmatic examples of NLS, $N$-wave eqs. Each such equation can be related to a RHP whose sewing function has specific dependence on the auxiliary 
parameters. For example, for these equations we have

$$
\begin{array}{llll}
N \text {-w } & i \frac{\partial G}{\partial x}-\lambda[J, G]=0, & i \frac{\partial G}{\partial t}-\lambda[I, G]=0, \\
\text { NLS } & i \frac{\partial G}{\partial x}-\lambda[J, G]=0, & i \frac{\partial G}{\partial t}-\lambda^{2}[J, G]=0
\end{array}
$$

where for the $N$-wave case the constant matrices $J$ and $I$ are diagonal ones with different eigenvalues. For the multicomponent NLS case the matrix $J$ is usually chosen as $J=\operatorname{diag}\left(\mathbb{1}_{k},-\mathbb{1}_{s}\right)$, where $k+s=n$ - the dimension of the matrices in the RHP. For the $N$-waves both $J=\operatorname{diag}\left(a_{1}, \ldots, a_{n}\right)$ and $I=\operatorname{diag}\left(b_{1}, \ldots, b_{n}\right)$ have different eigenvalues, i.e. $a_{j} \neq a_{k}$ and $b_{j} \neq b_{k}$ for $j \neq k$.

From now on we limit ourselves to the $N$-wave type NLEE.

The well known Zakharov-Shabat theorem [30, 31] states, that if $G(x, \lambda)$ satisfies the equations in (2.3) then the corresponding solution of the RHP will be common fundamental analytic solution of the operators:

$$
\begin{gathered}
L \xi^{ \pm}(x, t, \lambda) \equiv i \frac{\partial \xi^{ \pm}}{\partial x}+[J, Q(x, t)] \xi^{ \pm}(x, t, \lambda)-\lambda\left[J, \xi^{ \pm}(x, t, \lambda)\right]=0 . \\
M \xi^{ \pm}(x, t, \lambda) \equiv i \frac{\partial \xi^{ \pm}}{\partial t}+[I, Q(x, t)] \xi^{ \pm}(x, t, \lambda)-\lambda\left[I, \xi^{ \pm}(x, t, \lambda)\right]=0 .
\end{gathered}
$$

Thus to each specific $x$ or $t$ dependence of $G$ one can relate an ordinary differential operator like (2.5) and (2.6) whose potentials are determined through the same potential function $Q(x, t)=X_{1}(x, t)$. It is determined by the first non-trivial coefficient of $\xi^{ \pm}(x, t, \lambda)$ in its asymptotic expansion over the inverse powers of $\lambda$ :

$$
\begin{aligned}
\xi^{ \pm}(x, t, \lambda) & =\exp \left(\sum_{a=1}^{\infty} \lambda^{-a} X_{a}(x, t)\right) \\
& =\mathbb{1}+\lambda^{-1} X_{1}(x, t)+\cdots
\end{aligned}
$$

The pair of operators (2.5) and (2.6) provide the Lax pair for the $N$-wave eq., i.e., they commute, provided $Q(x, t)=X_{1}(x, t)$ satisfies:

$$
i\left[J, \frac{\partial Q}{\partial t}\right]-i\left[I, \frac{\partial Q}{\partial x}\right]-[[J, Q],[I, Q(x, t)]]=0
$$

It is obvious that we can choose $Q(x, t)$ so that its diagonal elements are 0. The next natural restrictions on $Q(x, t)$ is that it is a hermitian matrix:

$$
Q(x, t)=Q^{\dagger}(x, t)
$$

or B-hermitian:

$$
Q(x, t)=B Q^{\dagger}(x, t) B^{-1}, \quad B=\operatorname{diag}\left(\epsilon_{1}, \ldots, \epsilon_{n}\right),
$$

where $\epsilon_{j}= \pm 1$. In this way the $n \times n$ Lax operator $L$ allows us to solve the $N$-wave systems with $N=n(n-1) / 2$. In particular, for $n=3$ and 4 we get $N=3$ and 6 . 
One may use additional algebraic constraints on $Q(x, t)$, see [23, 9]. Important examples here are: i) the two wave equations $n=3$ and

$$
Q(x, t)=-A_{1} Q^{T}(x, t) A_{1}^{-1}, \quad A_{1}=\left(\begin{array}{ccc}
0 & 0 & 1 \\
0 & -1 & 0 \\
1 & 0 & 0
\end{array}\right),
$$

i.e. $Q_{12}=Q_{23}, Q_{21}=Q_{32}$;

ii) the four wave equations $n=4$ and

$$
Q(x, t)=-A_{2} Q^{T}(x, t) A_{2}^{-1}, \quad A_{2}=\left(\begin{array}{cccc}
0 & 0 & 0 & 1 \\
0 & 0 & 1 & 0 \\
0 & \epsilon & 0 & 0 \\
\epsilon & 0 & 0 & 0
\end{array}\right),
$$

i.e. $Q_{12}=-Q_{34}, Q_{13}=-\epsilon Q_{24}, Q_{14}=-\epsilon Q_{14}$ and $Q_{23}=-\epsilon Q_{23}$;

iii) the four wave equations can be obtained also with $n=5$ and

$$
Q(x, t)=-A_{3} Q^{T}(x, t) A_{3}^{-1}, \quad A_{3}=\left(\begin{array}{ccccc}
0 & 0 & 0 & 0 & 1 \\
0 & 0 & 0 & -1 & 0 \\
0 & 0 & 1 & 0 & 0 \\
0 & -1 & 0 & 0 & 0 \\
1 & 0 & 0 & 0 & 0
\end{array}\right)
$$

i.e. $Q_{12}=Q_{45}, Q_{13}=-Q_{35}, Q_{14}=Q_{25}, Q_{21}=Q_{54}, Q_{31}=-Q_{53}$ and $Q_{41}=Q_{52}$.

The reduction in ii) with $\epsilon=-1$ and the reduction iii) have simple algebraic meaning: they restrict $Q$ in ii) to be an element of the algebra $s p(4)$, while in the case iii) $Q \in s o(5)$. These two Lie algebras are isomorphic and therefore the corresponding 4 -wave equations are equivalent.

In [7] one can find a number of additional reductions of the $N$-wave equations.

\section{One-soliton solution and soliton decay}

\subsection{Generic one-solton solutions for $N$-waves}

From technical point of view the RHP may be viewed as the most convenient tool to solve the ISP for the corresponding Lax operator and then to construct the soliton solutions of the corresponding NLEE. We will start, using the dressing Zakharov-Shabat method [30,31] by constructing the one soliton solution of the $N$-wave equations.

We can always order the eigenvalues of $J$ so that $a_{1}>a_{2}>\cdots>a_{n}$, and also $\operatorname{tr} J=\sum_{s=1}^{n} a_{s}=0$. We assume also that similar constraints are valid for the matrix elements of $I$ : i.e. $b_{1}>b_{2}>\cdots>b_{n}$ and $\operatorname{tr} I=\sum_{s=1}^{n} b_{s}=0$.

Following [23] we write down the so-called dressing factor for the class of (2.9) reductions. The simplest of them have simple zeroes and poles in $\lambda$ which 
are characterized by a rank 1 projector:

$$
\begin{aligned}
u_{1}(x, t, \lambda) & =\mathbb{1}+\left(c_{1}(\lambda)-1\right) P_{1}, \quad P_{1}(x, t)=\frac{\left|n_{1}\right\rangle\left\langle n_{1}^{\dagger}\right|}{\left\langle n_{1}^{\dagger} \mid n_{1}\right\rangle}, \\
\left|n_{1}\right\rangle & =\xi_{0}^{+}\left(x, t, \lambda_{1}^{+}\right) e^{-i \lambda_{1}^{+} K}\left|n_{10}\right\rangle, \quad\left\langle n_{1}\right|=\left\langle n_{10}\right| e^{i \lambda_{1}^{-} K} \hat{\xi}_{0}^{-}\left(x, t, \lambda_{1}^{-}\right), \\
c_{1}(\lambda) & =\frac{\lambda-\lambda_{1}^{+}}{\lambda-\lambda_{1}^{-}}, \quad K=J x+I t, \quad \text { i.e. } \quad K_{s}=a_{s} x+b_{s} t .
\end{aligned}
$$

Here by $\xi_{0}^{ \pm}(x, t, \lambda)$ we mean regular solution of the RHP corresponding to the potential $Q_{(0)}(x, t)$ and $\hat{\xi}_{0}^{-}(x, t, \lambda)=\left(\xi^{-}\right)^{-1}(x, t, \lambda)$.

The new singular solution of the RHP:

$$
\begin{aligned}
& \xi_{1}^{+}(x, t, \lambda)=u_{1}(x, t, \lambda) \xi_{0}^{+}(x, t, \lambda) \hat{u}_{1}^{-}(\lambda), \\
& \xi_{1}^{-}(x, t, \lambda)=u_{1}(x, t, \lambda) \xi_{0}^{-}(x, t, \lambda) \hat{u}_{1}^{-}(\lambda),
\end{aligned}
$$

where $u_{1}^{-}(\lambda)=\lim _{x \rightarrow-\infty} u_{1}(x, t, \lambda)$ possess first order singularities at the points $\lambda_{1}^{ \pm}=\mu_{1} \pm i \nu_{1}$. They also solve eq. (2.5) but with another potential $Q_{(1)}(x, t)$. Taking the limit $\lambda \rightarrow \infty$ in (2.5) we find that $Q_{(1)}(x, t)$ is obtained from:

$$
Q_{(1)}(x, t)=Q_{0}(x, t)+\left(\lambda_{1}^{-}-\lambda_{1}^{+}\right)\left[J, P_{1}(x, t)\right] .
$$

Thus in order to evaluate $Q_{(1)}(x, t)$ all we need is to fix up the singular points $\lambda_{1}^{ \pm}$and the initial polarization vector $\left|n_{10}\right\rangle$, and to use the specific regular solution $\xi_{0}^{ \pm}(x, t, \lambda)$ of the RHP. In what follows we will use the simplest regular solution of the RHP corresponding to $Q_{(0)}(x, t)=0$, i.e. $\xi_{0}^{ \pm}(x, t, \lambda)=\mathbb{1}$ and $\chi_{0}^{ \pm}(x, t, \lambda) \equiv \xi_{0}^{ \pm}(x, t, \lambda) e^{-i \lambda K}=e^{-i \lambda K}$,

Analyzing this result one is able to discover a variety of different types of soliton solutions for the $N$-wave equations. They will be parametrized by the two new discrete eigenvalues $\lambda_{1}^{ \pm}$of $L$ and by the polarization vectors used to determine the projector $P_{1}$. The typical reduction gives $\lambda_{1}^{-}=\left(\lambda_{1}^{+}\right)^{*}$ and $P_{1}=$ $P_{1}^{\dagger}$. For the sake of simplicity we will limit ourselves only by rank-1 projectors; the more general cases of higher rank projectors and for classification of the soliton solutions see $[8,9]$

In what follows by one-soliton solution we will understand a matrix-valued solution $Q_{1 \mathrm{~s}}$ rather than the separate components $Q_{1 \mathrm{~s} ; \mathrm{jk}}$; the latter we will call the $(j, k)$-component of the soliton. From this point of view the generic onesoliton solution of the $N$-wave shows non-trivial interactions [20, 23] which can be viewed as soliton decay or soliton fusion. In particular on figure 1 taking $n=3$, we see the process in which the $(1,3)$ soliton component decays into $(1,2)$ and $(2,3)$ components. Choosing other initial conditions we may have also the alternative process of soliton fusion in which the components $(1,2)$ and $(2,3)$ generate the $(1,3)$ component. 


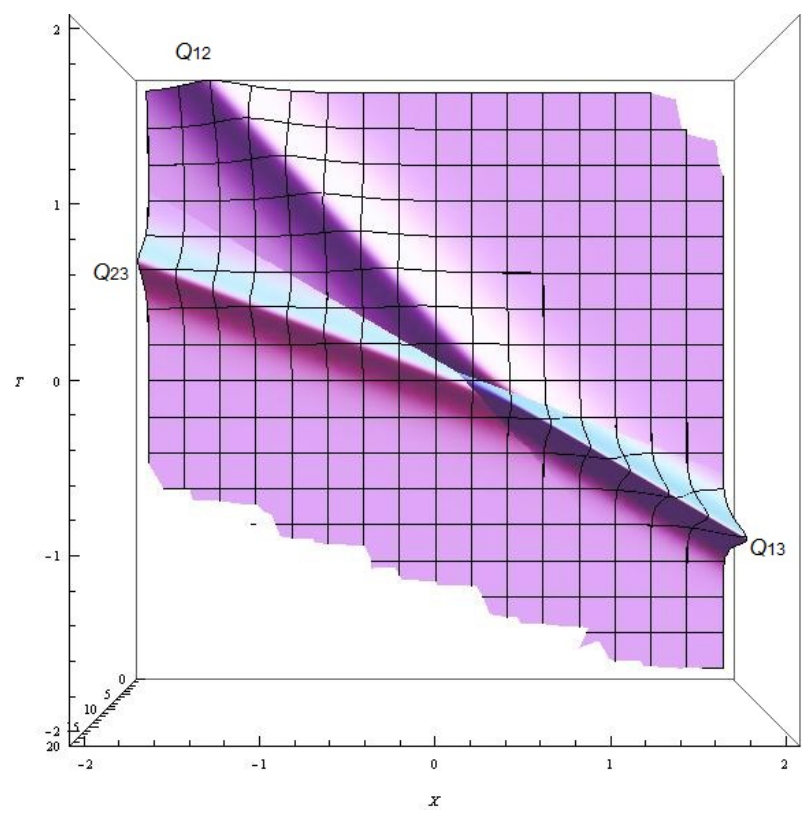

Figure 1: Generic one-soliton solution of 3-wave interactions. Each of the functions $\left|Q_{j k}\right|$ plotted has a maximum along a semi-line, see eq. (3.7)

Indeed, the generic one-soliton solution for the $N$-wave system is given by:

$$
\begin{aligned}
Q_{1 \mathrm{~s} ; \mathrm{jk}}(x, t) & =-\frac{2 i \nu_{1}\left(a_{j}-a_{k}\right) n_{1 ; k} n_{1 ; j}^{*}}{\left\langle n_{1}^{\dagger} \mid n_{1}\right\rangle} \\
& =-\frac{2 i \nu_{1}\left(a_{j}-a_{k}\right) \exp \left(i\left(\mu_{1}\left(K_{k}-K_{j}\right)+\phi_{10 ; k}-\phi_{10 ; j}\right)\right.}{\sum_{s=1}^{n} \exp \left(\nu_{1}\left(2 K_{s}-K_{j}-K_{k}\right)+2 \xi_{10, s}-\xi_{10, j}-\xi_{10, k}\right)} \\
& =-\frac{i \nu_{1}\left(a_{j}-a_{k}\right) e^{-i\left(\phi_{1 ; j}-\phi_{1 ; k}\right)}}{\cosh \left(\xi_{1 ; j}-\xi_{1 ; k}\right)+\sum_{s \neq k, j} \frac{1}{2} \exp \left(2 \xi_{1 ; s}-\xi_{1 ; j}-\xi_{1 ; k}\right)} .
\end{aligned}
$$

where we have assumed that all components of the polarization vector $\left|n_{10}\right\rangle$ are non-vanishing $n_{10 ; k}=\exp \left(\xi_{10 ; k}+i \phi_{10 ; k}\right)$ and

$$
\xi_{1 ; j}(x, t)=\nu_{1} K_{j}(x, t)-\ln \left|n_{10 ; j}\right|, \quad \phi_{1 ; j}(x, t)=\mu_{1} K_{j}+\phi_{10 ; j} .
$$


For $n=3$ we have:

$$
\begin{aligned}
Q_{1 \mathrm{~s} ; 12}(x, t) & =\frac{-i \nu_{1}\left(a_{1}-a_{2}\right) e^{-i\left(\phi_{1 ; 1}-\phi_{1 ; 2}\right)}}{\cosh \left(\xi_{1,1}-\xi_{1,2}\right)+\frac{1}{2} e^{2 \xi_{1,3}-\xi_{1,1}-\xi_{1,2}}} \\
Q_{1 \mathrm{~s} ; 13}(x, t) & =\frac{-i \nu_{1}\left(a_{1}-a_{3}\right) e^{-i\left(\phi_{1 ; 1}-\phi_{1 ; 3}\right)}}{\cosh \left(\xi_{1,1}-\xi_{1,3}\right)+\frac{1}{2} e^{2 \xi_{1,2}-\xi_{1,1}-\xi_{1,3}}} \\
Q_{1 \mathrm{~s} ; 23}(x, t) & =\frac{-i \nu_{1}\left(a_{2}-a_{3}\right) e^{-i\left(\phi_{1 ; 2}-\phi_{1 ; 3}\right)}}{\cosh \left(\xi_{1,2}-\xi_{1,3}\right)+\frac{1}{2} e^{2 \xi_{1,1}-\xi_{1,2}-\xi_{1,3}}} .
\end{aligned}
$$

where we can also assume that $\xi_{1,1}+\xi_{1,2}+\xi_{1,3}=0$. Following Zakharov and Manakov [20] we analyze the asymptotic behavior of the different components of the generic one-soliton solution for $x \rightarrow \infty$. The results are, see fig. 1:

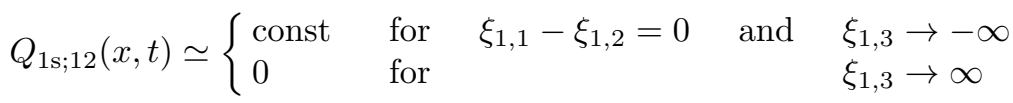

$$
\begin{aligned}
& Q_{1 \mathrm{~s} ; 13}(x, t) \simeq\left\{\begin{array}{lll}
\text { const } & \text { for } \\
0 & \text { for }
\end{array} \xi_{1,1}-\xi_{1,3}=0 \quad \text { and } \quad \begin{array}{l}
\xi_{1,2} \rightarrow-\infty \\
\xi_{1,2} \rightarrow \infty
\end{array}\right.
\end{aligned}
$$

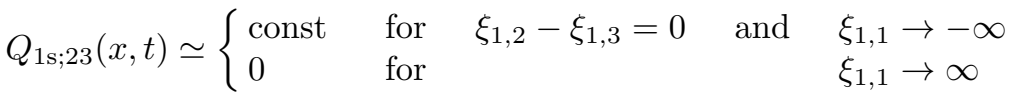

Thus we find that for $t \rightarrow-\infty$ only the $(1,3)$ component of the soliton is nontrivial, which then decays into the $(1,2)$ and $(2,3)$ components.

Note that in this respect the $N$-wave equations differ from the other soliton equations (e.g., NLS, s-G, KdV, MKdV and many others) with nontrivial dispersion laws which have stable one-soliton solutions.

\subsection{Additional reductions and new types of solitons}

As we mentioned above the reductions iii) and reduction ii) with $\epsilon=-1$ lead to the following 4-wave equations [23]:

$$
\begin{aligned}
i\left(a_{1}-a_{2}\right) Q_{12 ; t}-i\left(b_{1}-b_{2}\right) Q_{12 ; x}-\kappa Q_{13} Q_{23}^{*}(x, t) & =0, \\
i a_{1} Q_{13 ; t}-i b_{1} Q_{13 ; x}-\kappa\left(Q_{12} Q_{23}(x, t)-Q_{14} Q_{23}^{*}\right) & =0, \\
i\left(a_{1}+a_{2}\right) Q_{14 ; t}-i\left(b_{1}+b_{2}\right) Q_{14 ; x}-\kappa Q_{13} Q_{23}(x, t) & =0, \\
i a_{2} Q_{23 ; t}-i b_{2} Q_{23 ; x}+\kappa\left(Q_{14} Q_{13}^{*}(x, t)+Q_{13} Q_{12}^{*}\right) & =0,
\end{aligned}
$$

where $\kappa=b_{2} a_{1}-a_{2} b_{1}$.

$$
Q(x, t)=-A_{3} Q^{T}(x, t) A_{3}^{-1}, \quad Q=\left(\begin{array}{ccccc}
0 & Q_{12} & Q_{13} & Q_{14} & 0 \\
Q_{12}^{*} & 0 & Q_{23} & 0 & Q_{14} \\
Q_{13}^{*} & Q_{23}^{*} & 0 & Q_{23} & -Q_{13} \\
Q_{14}^{*} & 0 & Q_{23}^{*} & 0 & Q_{12} \\
0 & Q_{14}^{*} & -Q_{13}^{*} & Q_{12}^{*} & 0
\end{array}\right)
$$


The integrability of the 4-wave equations and their physical applications were discovered by Manakov and Zakharov, see [23]. The derivation of their soliton solutions was investigated in [14], see also [11, 10].

In order to apply the dressing method to the systems with other reductions it has to be modified. In particular, the dressing factor must be invariant with respect to the reduction. This can be achieved as follows:

$$
\begin{aligned}
u_{1}(x, t, \lambda) & =\mathbb{1}+\left(c_{1}(\lambda)-1\right) P_{1}+\left(c_{1}^{-1}(\lambda)-1\right) \bar{P}_{1}, \quad P_{1}(x, t)=\frac{\left|n_{1}\right\rangle\left\langle n_{1}^{\dagger}\right|}{\left\langle n_{1}^{\dagger} \mid n_{1}\right\rangle}, \\
\left|n_{1}\right\rangle & =\xi_{0}^{+}\left(x, t, \lambda_{1}^{+}\right) e^{-i \lambda_{1}^{+} K}\left|n_{10}\right\rangle, \quad\left\langle n_{1}\right|=\left\langle n_{10}\right| e^{i \lambda_{1}^{-} K} \hat{\xi}_{0}^{-}\left(x, t, \lambda_{1}^{-}\right), \\
c_{1}(\lambda) & =\frac{\lambda-\lambda_{1}^{+}}{\lambda-\lambda_{1}^{-}}, \quad \bar{P}_{1}(x, t)=A_{3} P_{1}^{T}(x, t) A_{3}^{-1}
\end{aligned}
$$

where $A_{3}$ is the matrix that determine the reductions in eq. (2.13) and the constant polarization vector $\left|n_{10}\right\rangle$ satisfies the constraint $\left\langle n_{10}\left|A_{3}\right| n_{10}\right\rangle$. The corresponding soliton solutions are obtained through:

$$
\left[J, Q_{1 \mathrm{~s}}\right]=\left(\lambda_{1}^{-}-\lambda_{1}^{+}\right)\left[J, P_{1}(x, t)-\bar{P}_{1}(x, t)\right] .
$$

Similar technique can be used also for the other reductions listed in Section 2.

Below we will use the following parametrization of the polarization vector $\left|n_{10}\right\rangle$, that are compatible with the constraint $\left\langle n_{10}\left|A_{3}\right| n_{10}\right\rangle=0$ and with the additional restriction $n_{10,1}=n_{10,5}^{*}, n_{10,2}=n_{10,4}^{*}$ and $n_{10,3}=\sqrt{2}$ :

$n_{10,1}=n_{10,5}^{*}=\sinh \theta_{0} e^{i \phi_{10,1}}, \quad n_{10,2}=n_{10,4}^{*}=\cosh \theta_{0} e^{i \phi_{10,2}}, \quad n_{10,3}=\sqrt{2}$.

As a result we get the following generic one-soliton solution:

$$
\begin{aligned}
Q_{12}(x, t) & =\frac{i \nu_{1} \sinh 2 \theta_{0}}{\Delta_{1}(x, t)} \cosh \left(\nu_{1}\left(K_{1}+K_{2}\right)\right) e^{-i \mu_{1}\left(K_{1}-K_{2}\right)+i\left(\phi_{10,1}-\phi_{10,2}\right)}, \\
Q_{13}(x, t) & =-\frac{2 i \sqrt{2} \nu_{1} \sinh \theta_{0}}{\Delta_{1}(x, t)} \sinh \left(\nu_{1} K_{1}\right) e^{-i \mu_{1} K_{1}+i \phi_{10,1},}, \\
Q_{14}(x, t) & =\frac{i \nu_{1} \sinh 2 \theta_{0}}{\Delta_{1}(x, t)} \cosh \left(\nu_{1}\left(K_{1}-K_{2}\right)\right) e^{-i \mu_{1}\left(K_{1}+K_{2}\right)+i\left(\phi_{10,1}+\phi_{10,2}\right)}, \\
Q_{23}(x, t) & =\frac{2 i \sqrt{2} \nu_{1} \cosh \theta_{0}}{\Delta_{1}(x, t)} \cosh \left(\nu_{1} K_{2}\right) e^{-i \mu_{1} K_{2}+i \phi_{10,2}},
\end{aligned}
$$

where

$$
\Delta_{1}(x, t)=2\left(\sinh ^{2} \theta_{0} \cosh \left(2 \nu_{1} K_{1}\right)+\cosh ^{2} \theta_{0} \cosh \left(2 \nu_{1} K_{2}\right)+1\right) .
$$

\subsection{Doublet solitons}

Let the action of $\mathbb{Z}_{2} \times \mathbb{Z}_{2}$ in the space of fundamental solutions of the linear problem is given by $[10,11]$ :

$$
\chi^{-}(x, t, \lambda)=\left(\left(\chi^{+}\right)^{\dagger}\left(x, t, \lambda^{*}\right)\right)^{-1}, \quad \chi^{-}(x, t, \lambda)=\left(\left(\chi^{+}\right)^{T}(x, t,-\lambda)\right)^{-1},
$$


Consequently the potential $U(x, t, \lambda)$ satisfies the following symmetry conditions

$$
U^{\dagger}\left(x, t, \lambda^{*}\right)=U(x, t, \lambda), \quad U^{T}(x, t,-\lambda)=-U(x, t, \lambda) .
$$

Then, in the case of the so(5) algebra we put

$$
Q_{j k}(x, t)=i q_{j k}(x, t) .
$$

with $q_{j k}(x, t)$ being real valued fields and get the following $\mathbb{Z}_{2} \times \mathbb{Z}_{2}$-reduced 4-wave system

$$
\begin{aligned}
& \left(a_{1}-a_{2}\right) q_{12, t}(x, t)-\left(b_{1}-b_{2}\right) q_{12, x}(x, t)+\kappa q_{13}(x, t) q_{23}(x, t)=0 \\
& a_{1} q_{13, t}(x, t)-b_{1} q_{13, x}(x, t)+\kappa\left(q_{14}(x, t)-q_{12}(x, t)\right) q_{23}(x, t)=0 \\
& \left(a_{1}+a_{2}\right) q_{14, t}(x, t)-\left(b_{1}+b_{2}\right) q_{14, x}(x, t)-\kappa q_{13}(x, t) q_{23}(x, t)=0 \\
& a_{2} q_{23, t}(x, t)-b_{2} q_{23, x}(x, t)+\kappa\left(q_{12}(x, t)+q_{14}(x, t)\right) q_{13}(x, t)=0
\end{aligned}
$$

where $\kappa=a_{1} b_{2}-a_{2} b_{1}$.

In accordance with what we said above the dressing factor $u(x, t, \lambda)$ must be invariant under the action of $\mathbb{Z}_{2} \times \mathbb{Z}_{2}$, i.e.

$$
\left(u^{\dagger}\left(x, t, \lambda^{*}\right)\right)^{-1}=u(x, t, \lambda), \quad\left(u^{T}(x, t,-\lambda)\right)^{-1}=u(x, t, \lambda) .
$$

Next we find that the poles of the dressing matrix can be purely imaginary, i.e.

$$
\lambda_{1}^{ \pm}= \pm i \nu_{1}, \quad \nu_{1}>0
$$

Thus the invariance condition implies that the dressing matrix gets the form

$$
u(x, t, \lambda)=\mathbb{1}+\frac{A(x, t)}{\lambda-i \nu_{1}}+\frac{A_{3} A^{*}(x, t) A_{3}}{\lambda+i \nu_{1}}, \quad A^{*}(x, t)=-A(x, t) .
$$

where $A_{3}$ was introduced in eq. (2.13). Following already discussed procedures we derive in the simplest case that the explicit form of $A(x, t)$ is

$$
A(x, t)=\frac{2 i \nu_{1}|n(x, t)\rangle\langle n(x, t)|}{\langle n(x, t) \mid n(x, t)\rangle}
$$

where the vector $|n(x, t)\rangle=e^{-\nu_{1}(J x+I t)}\left|n_{10}\right\rangle$ is real. Consequently the soliton solution written in a standard matrix notation is

$$
Q_{k l}(x, t)=\left(1-\delta_{k l}\right) \frac{2 i \nu_{1}\left(n_{k}(x, t) n_{l}(x, t)-(-1)^{k+l} n_{6-k}(x, t) n_{6-l}(x, t)\right)}{\langle n(x, t) \mid n(x, t)\rangle}
$$

In what follows it will be appropriate to use somewhat different parametrization for the polarization vector:

$$
n_{10,1}=e^{\delta_{1}} \sinh \theta_{0}, \quad n_{10,2}=e^{\delta_{2}} \cosh \theta_{0}, \quad n_{10,3}=\sqrt{2},
$$




$$
n_{10,4}=e^{-\delta_{2}} \cosh \theta_{0}, \quad n_{10,5}=e^{-\delta_{1}} \sinh \theta_{0} .
$$

Then the corresponding doublet soliton solution of the 4-wave system (3.15) takes the form

$$
\begin{aligned}
& q_{12}(x, t)=\frac{\nu_{1} \sinh 2 \theta_{0}}{\Delta_{2}} \cosh \left(\nu_{1}\left(K_{1}+K_{2}\right)+\delta_{1}+\delta_{2}\right), \\
& q_{13}(x, t)=-\frac{2 \sqrt{2} \nu_{1} \sinh \theta_{0}}{\Delta_{2}} \sinh \left(\nu_{1} K_{1}+\delta_{1}\right), \\
& q_{14}(x, t)=\frac{\nu_{1} \sinh 2 \theta_{0}}{\Delta_{2}} \cosh \left(\nu_{1}\left(K_{1}-K_{2}\right)+\delta_{1}-\delta_{2}\right), \\
& q_{23}(x, t)=\frac{2 \sqrt{2} \nu_{1}}{\Delta_{2}} \cosh \left(\nu_{1} K_{2}+\delta_{2}\right), \\
& \Delta_{2}(x, t)=\sinh ^{2} \theta_{0} \cosh 2\left(\nu_{1} K_{1}+\delta_{1}\right)+\cosh ^{2} \theta_{0} \cosh 2\left(\nu_{1} K_{2}+\delta_{2}\right)+1,
\end{aligned}
$$

where

$$
\delta_{1}=\frac{1}{2} \ln \frac{n_{10,5}}{n_{10,1}}, \quad \delta_{2}=\frac{1}{2} \ln \frac{n_{10,4}}{n_{10,2}} .
$$

In particular when $n_{10,1}=n_{10,5}$ and $n_{10,2}=n_{10,4}$ or in other words $\delta_{1}=\delta_{2}=0$ we obtain

$$
\begin{gathered}
q_{12}(x, t)=\frac{\nu_{1} \sinh \left(2 \theta_{0}\right)}{\Delta_{D}} \cosh \left[\nu_{1}\left(K_{1}+K_{2}\right)\right], \\
q_{13}(x, t)=-\frac{2 \sqrt{2} \nu_{1} \sinh \theta_{0}}{\Delta_{D}} \sinh \left(\nu_{1} K_{1}\right), \\
q_{14}(x, t)=\frac{\nu_{1} \sinh \left(2 \theta_{0}\right)}{\Delta_{D}} \cosh \left[\nu_{1}\left(K_{1}-K_{2}\right)\right], \\
q_{23}(x, t)=\frac{2 \sqrt{2} \nu_{1} \cosh \theta_{0}}{\Delta_{D}} \cosh \left(\nu_{1} K_{2}\right),
\end{gathered}
$$

where $\theta_{0} \in \mathbb{R}$ and

$$
\Delta_{D}(x, t)=\sinh ^{2} \theta_{0} \sinh ^{2}\left(\nu_{1} K_{1}\right)+\cosh ^{2} \theta_{0} \cosh ^{2}\left(\nu_{1} K_{2}\right) .
$$

\subsection{Quadruplet solitons}

There is another way to ensure the $\mathbb{Z}_{2} \times \mathbb{Z}_{2}$ invariance of the dressing factor. This time we consider dressing factors $g(x, t, \lambda)$ with two more poles. The requirements (3.19) lead to the following dressing matrix

$$
u(x, t, \lambda)=\mathbb{1}+\frac{A(x, t)}{\lambda-\lambda_{1}^{+}}+\frac{S_{0} A^{*}(x, t) S_{0}}{\lambda-\left(\lambda_{1}^{+}\right)^{*}}-\frac{S_{0} A(x, t) S_{0}}{\lambda+\lambda_{1}^{+}}-\frac{A^{*}(x, t)}{\lambda+\left(\lambda_{1}^{+}\right)^{*}} .
$$

The corresponding soliton solution takes the form:

$$
[J, Q(x, t)]=\left[J, A+S_{0} A^{*} S_{0}-S_{0} A S_{0}-A^{*}(x, t)\right] .
$$


Like in previous considerations we decompose the matrix $A(x, t)$ into the factors

$$
A(x, t)=X(x, t) F^{T}(x, t),
$$

where:

$$
\begin{aligned}
F(x, t) & =e^{i \lambda_{1}^{+} K}\left|n_{10}\right\rangle=\left(F_{1}, F_{2}, F_{3}, F_{4}, F_{5}\right)^{T} & & \\
F_{1,5} & =e^{\mp \kappa_{1} \pm i \Phi_{1}}, & & F_{2,4}=e^{\mp \kappa_{2} \pm i \Phi_{2}}, \quad F_{3}=\sqrt{2}, \\
\kappa_{1,2} & =\nu_{1} K_{1,2}+\delta_{1,2}, & & \Phi_{1,2}=\mu_{1} K_{1,2}+i \phi_{10 ; 1,2} .
\end{aligned}
$$

For the factor $X(x, t)$ we derive an algebraic system of equations (see e.g. $[9,11])$ whose solution is:

$$
X(x, t)=\frac{1}{\Delta(x, t)}\left(a^{*}(x, t) F+b(x, t) F^{*}-c(x, t) S_{0} F^{*}\right)
$$

where

$$
\begin{aligned}
a(x, t) & =\frac{F^{T} F}{\mu_{1}+i \nu_{1}}, \quad b(x, t)=\frac{F^{T} S_{0} F^{*}}{\nu_{1}}, \quad c(x, t)=\frac{F^{T} F^{*}}{\mu_{1}}, \\
\Delta(x, t) & =|a(x, t)|^{2}-b^{2}(x, t)-c^{2} .
\end{aligned}
$$

Inserting the explicit form of $F$ from eq. (3.25) we get:

$$
\begin{aligned}
a & =\frac{\mu_{1} a_{0}-\nu_{1} a_{1}}{\mu_{1}^{2}+\nu_{1}^{2}}-i \frac{\nu_{1} a_{0}+\mu_{1} a_{1}}{\mu_{1}^{2}+\nu_{1}^{2}}, \\
a_{0} & =\cosh ^{2} \theta_{0} \cosh \left(2 \kappa_{2}\right) \cos \left(2 \Phi_{2}\right)+\sinh ^{2} \theta_{0} \cosh \left(2 \kappa_{1}\right) \cos \left(2 \Phi_{1}\right)+1, \\
a_{1} & =\cosh ^{2} \theta_{0} \sinh \left(2 \kappa_{2}\right) \sin \left(2 \Phi_{2}\right)+\sinh ^{2} \theta_{0} \sinh \left(2 \kappa_{1}\right) \sin \left(2 \Phi_{1}\right), \\
b & =\frac{2}{\nu_{1}}\left(\cosh ^{2} \theta_{0} \cosh \left(\kappa_{1}+\kappa_{2}\right) \cosh \left(\kappa_{1}-\kappa_{2}\right)-\sinh ^{2} \kappa_{1}\right), \\
c & =-\frac{1}{\mu_{1}}\left(\sinh ^{2} \theta_{0} \cos \left(2 \Phi_{1}\right)-\cosh ^{2} \kappa_{1} \cos \left(2 \Phi_{2}\right)\right) .
\end{aligned}
$$

Inserting the result for $X(x, t)$ and $F(x, t)$ in (3.23) we obtain the quadruplet (or breather-like) solution

$$
\begin{aligned}
& {\left[J, Q_{\mathrm{br}}(x, t)\right]=\frac{1}{\Delta}\left[J, C(x, t)-A_{3} C^{T}(x, t) A_{3}\right], } \\
& C(x, t)=a^{*} F F^{T}(x, t)-a F^{*} F^{\dagger}(x, t)-i b\left(F^{*} F^{T}(x, t)+F F^{\dagger}(x, t)\right) \\
&+c\left(F^{*} F^{T}(x, t) A_{3}+A_{3} F F^{\dagger}(x, t)\right),
\end{aligned}
$$

Due to the reductions we have $Q_{\mathrm{br} ; \mathrm{jk}}=i q_{\mathrm{br} ; \mathrm{jk}}$ with real valued functions $q_{\mathrm{br} ; \mathrm{jk}}$ 
given by:

$$
\begin{aligned}
q_{\mathrm{br} ; 12}(x, t)=- & \frac{2 \sinh \left(2 \theta_{0}\right)}{\Delta}\left(\left(c \sinh \left(\kappa_{1}-\kappa_{2}\right)+a_{0} \sinh \left(\kappa_{1}+\kappa_{2}\right)\right) \sin \left(\Phi_{1}+\Phi_{2}\right)\right. \\
& \left.+\left(a_{1} \cos \left(\Phi_{1}+\Phi_{2}\right)-b \cos \left(\Phi_{1}-\Phi_{2}\right)\right) \cosh \left(\kappa_{1}+\kappa_{2}\right)\right), \\
q_{\mathrm{br} ; 13}(x, t)= & \frac{4 \sqrt{2} \sinh \left(\theta_{0}\right)}{\Delta}\left(\left(a_{0}-c\right) \cosh \kappa_{1} \sin \Phi_{1}+\left(a_{1}-b\right) \cosh \kappa_{1} \cos \Phi_{1}\right), \\
q_{\mathrm{br} ; 14}(x, t)=- & \frac{2 \sinh \left(2 \theta_{0}\right)}{\Delta}\left(\left(c \sinh \left(\kappa_{1}+\kappa_{2}\right)+a_{0} \sinh \left(\kappa_{1}-\kappa_{2}\right)\right) \sin \left(\Phi_{1}-\Phi_{2}\right)\right. \\
& \left.+\left(b \cos \left(\Phi_{1}+\Phi_{2}\right)-a_{1} \cos \left(\Phi_{1}-\Phi_{2}\right)\right) \cosh \left(\kappa_{1}-\kappa_{2}\right)\right), \\
q_{\mathrm{br} ; 23}(x, t)= & \frac{4 \sqrt{2} \cosh \left(\theta_{0}\right)}{\Delta}\left(\left(c-a_{0}\right) \sinh \kappa_{2} \sin \Phi_{2}+\left(b-a_{1}\right) \cosh \kappa_{2} \cos \Phi_{2}\right),
\end{aligned}
$$

\section{Two-soliton solutions and soliton interactions}

Let us now construct the two-soliton solutions for the 3-wave equations by applying twice the dressing procedure. We can do this in two alternative ways, but the final results is independent of the order in which we apply the dressing.

$$
u_{2 \mathrm{~s} ; \mathrm{A}}=u_{2,1}(x, t, \lambda) u_{1}(x, t, \lambda), \quad u_{2 \mathrm{~s} ; \mathrm{B}}=u_{1,2}(x, t, \lambda) u_{2}(x, t, \lambda),
$$

where

and

$$
\begin{aligned}
u_{j}(x, t, \lambda) & =\mathbb{1}+\left(c_{j}(\lambda)-1\right) P_{j}, \quad c_{j}(\lambda)=\frac{\lambda-\lambda_{j}^{+}}{\lambda-\lambda_{j}^{-}}, \\
P_{j}(x, t) & =\frac{\left|n_{j}\right\rangle\left\langle n_{j}^{\dagger}\right|}{\left\langle n_{j}^{\dagger} \mid n_{j}\right\rangle},
\end{aligned}
$$

$$
u_{2,1}(x, t, \lambda)=\mathbb{1}+\left(c_{2}(\lambda)-1\right) \mathbf{P}_{2,1}, \quad u_{1,2}(x, t, \lambda)=\mathbb{1}+\left(c_{1}(\lambda)-1\right) \mathbf{P}_{1,2},
$$

Here

$$
\begin{aligned}
P_{2,1}(x, t) & =\frac{\left|\mathbf{n}_{2}\right\rangle\left\langle\mathbf{n}_{2}^{\dagger}\right|}{\left\langle\mathbf{n}_{2}^{\dagger} \mid \mathbf{n}_{2}\right\rangle}, & P_{1,2}(x, t) & =\frac{\left|\mathbf{n}_{1}\right\rangle\left\langle\mathbf{n}_{1}^{\dagger}\right|}{\left\langle\mathbf{n}_{1}^{\dagger} \mid \mathbf{n}_{1}\right\rangle}, \\
\left|\mathbf{n}_{1}\right\rangle & =u_{2}\left(x, t, \lambda_{1}^{+}\right)\left|n_{1}\right\rangle, & \left|\mathbf{n}_{2}\right\rangle & =u_{1}\left(x, t, \lambda_{2}^{+}\right)\left|n_{2}\right\rangle,
\end{aligned}
$$

where

$$
\begin{aligned}
\left|\mathbf{n}_{1}\right\rangle & =\hat{u}_{2}^{-}\left(\lambda_{1}^{+}\right)\left|n_{1}\right\rangle+\left(c_{2}\left(\lambda_{1}^{+}\right)-1\right) \frac{\kappa_{21}}{\kappa_{22}} \hat{u}_{2}^{-}\left(\lambda_{1}^{+}\right)\left|n_{2}\right\rangle, \\
\left|\mathbf{n}_{2}\right\rangle & =\hat{u}_{1}^{-}\left(\lambda_{2}^{+}\right)\left|n_{2}\right\rangle+\left(c_{1}\left(\lambda_{2}^{+}\right)-1\right) \frac{\kappa_{12}}{\kappa_{11}} \hat{u}_{1}^{-}\left(\lambda_{2}^{+}\right)\left|n_{1}\right\rangle, \\
\left\langle\mathbf{n}_{2}^{\dagger} \mid \mathbf{n}_{2}\right\rangle & =\frac{4 \nu_{1} \nu_{2}}{\kappa_{11}} Z, \quad\left\langle\mathbf{n}_{1}^{\dagger} \mid \mathbf{n}_{1}\right\rangle=\frac{4 \nu_{1} \nu_{2}}{\kappa_{22}} Z, \\
Z(x, t) & =\left|\lambda_{2}^{+}-\lambda_{1}^{-}\right|^{2} \kappa_{11} \kappa_{22}-4 \nu_{1} \nu_{2} \kappa_{12} \kappa_{21}, \quad \kappa_{12}=\left\langle n_{j}^{\dagger}\left|\hat{u}_{1}^{-}\left(\lambda_{2}^{+}\right)\right| n_{k}\right\rangle
\end{aligned}
$$


The corresponding two-soliton solution is given by:

$$
\begin{aligned}
Q_{2 \mathrm{~s}}(x, t) & =\left(\lambda_{1}^{-}-\lambda_{1}^{+}\right)\left[J, P_{1}\right]+\left(\lambda_{1}^{-}-\lambda_{1}^{+}\right)\left[J, \mathbf{P}_{2}\right] \\
& =-2 i\left[J, \nu_{1} \mathbf{P}_{1}+\nu_{2} P_{2}\right] .
\end{aligned}
$$

These two results provide the same expression for the two-soliton solution and are behind the nonlinear superposition principle for the Bäcklund transformations $[3,12]$. Skipping the details the generic two-soliton solution takes the form:

$$
\begin{aligned}
Q_{2 \mathrm{~s}}(x, t) & =-\frac{2 i}{Z(x, t)}[J, C(x, t)], \\
C(x, t) & =\left|\lambda_{2}^{+}-\lambda_{1}^{-}\right|^{2}\left(\nu_{1} \kappa_{22}\left|n_{1}\right\rangle\left\langle n_{1}^{\dagger}\left|+\nu_{2} \kappa_{11}\right| n_{2}\right\rangle\left\langle n_{2}^{\dagger}\right|\right) \\
& -2 i \nu_{1} \nu_{2}\left(\lambda_{2}^{-}-\lambda_{1}^{+}\right) \kappa_{12}\left|n_{1}\right\rangle\left\langle n_{2}^{\dagger}\left|+2 i \nu_{1} \nu_{2}\left(\lambda_{2}^{+}-\lambda_{1}^{-}\right) \kappa_{21}\right| n_{2}\right\rangle\left\langle n_{1}^{\dagger}\right| .
\end{aligned}
$$

It is of interest also to present the two-soliton solutions with particular choices for their polarization vectors. If we choose:

$$
\left|n_{10}\right\rangle=\left(\begin{array}{c}
n_{10,1} \\
0 \\
n_{10,3}
\end{array}\right), \quad\left|n_{20}\right\rangle=\left(\begin{array}{c}
n_{20,1} \\
n_{20,2} \\
0
\end{array}\right)
$$

and insert them into eq. (4.7) we obtain:

$$
\begin{aligned}
& \tilde{Z}(x, t)=\left\{e^{\nu_{1}\left(K_{1}-K_{3}\right)+\xi_{10,1}-\xi_{10,3}} \cosh \left(\nu_{1}\left(K_{1}-K_{2}\right)+\gamma_{1}\right)\right. \\
& \left.+\left|\frac{\lambda_{2}^{+}-\lambda_{1}^{-}}{\lambda_{2}^{+}-\lambda_{1}^{+}}\right| e^{-\nu_{1}\left(K_{1}-K_{3}\right)} \cosh \left(\nu_{2}\left(K_{1}-K_{2}\right)+\gamma_{2}\right)\right\} \\
& \gamma_{1}=\ln \left|\frac{\lambda_{2}^{+}-\lambda_{1}^{-}}{\lambda_{2}^{+}-\lambda_{1}^{+}}\right|+\xi_{20,1}-\xi_{20,2}, \quad \gamma_{2}=\xi_{10,1}-\xi_{10,3}+\xi_{20,1}-\xi_{20,2}, \\
& Q_{2 \mathrm{~s} ; 12}(x, t)=-\frac{i\left(a_{1}-a_{2}\right)\left(\lambda_{2}^{-}-\lambda_{1}^{+}\right) \nu_{2}}{\tilde{Z}(x, t)} e^{-i \mu_{1}\left(K_{1}-K_{2}\right)+i\left(\phi_{20,1}-\phi_{20,2}\right)} \\
& \left(\left(\lambda_{2}^{+}-\lambda_{1}^{+}\right) e^{\nu_{1}\left(K_{1}-K_{3}\right)+\xi_{10,1}-\xi_{10,3}}+\left(\lambda_{2}^{+}-\lambda_{1}^{-}\right) e^{-\nu_{1}\left(K_{1}-K_{3}\right)-\xi_{10,1}+\xi_{10,3}}\right), \\
& Q_{2 \mathrm{~s} ; 13}(x, t)=-\frac{i\left(a_{1}-a_{3}\right)\left(\lambda_{2}^{-}-\lambda_{1}^{+}\right) \nu_{1}}{\tilde{Z}(x, t)} e^{-i \mu_{1}\left(K_{1}-K_{3}\right)+i\left(\phi_{10,1}-\phi_{10,3}\right)} \\
& \left(\left(\lambda_{2}^{-}-\lambda_{1}^{+}\right) e^{\nu_{2}\left(K_{1}-K_{2}\right)+\xi_{20,1}-\xi_{20,2}}+\left(\lambda_{2}^{-}-\lambda_{1}^{+}\right) e^{-\nu_{2}\left(K_{1}-K_{2}\right)-\xi_{20,1}+\xi_{20,2}}\right),
\end{aligned}
$$




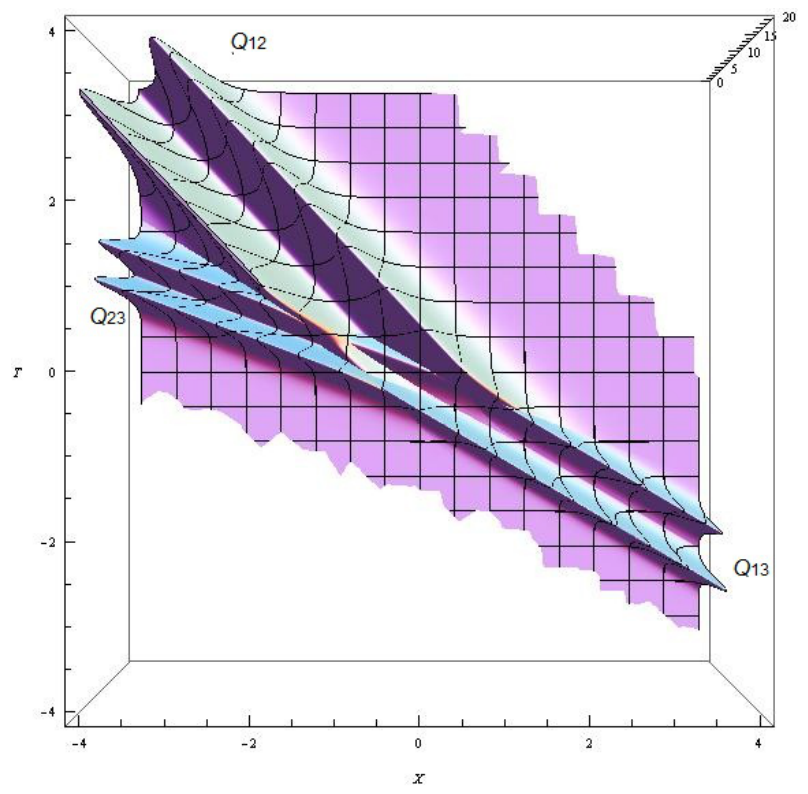

Figure 2: Generic two-soliton solution of 3-wave interactions. Now each of the functions $\left|Q_{j k}\right|$ plotted has two maxima along the same semi-lines as in fig. 1.

$$
\begin{aligned}
& Q_{2 \mathrm{~s} ; 23}(x, t)=-\frac{i\left(a_{2}-a_{3}\right)\left(\lambda_{2}^{+}-\lambda_{1}^{-}\right) \nu_{1} \nu_{2}}{\tilde{Z}(x, t)} \\
& \quad \times \exp \left(-i \mu_{1}\left(K_{1}-K_{3}\right)+i \mu_{2}\left(K_{1}-K_{2}\right)+i\left(\phi_{10,1}-\phi_{20,1}+\phi_{20,2}-\phi_{10,3}\right)\right)
\end{aligned}
$$

Next we can evaluate the asymptotics of the two-soliton solution for large $t$ and $x$ and describe the interaction of solitons. However in the $N$-wave case the velocities of the solitons of type $(j, k)$ are all equal to the corresponding group velocity $v_{j k}=\left(a_{j}-a_{k}\right) /\left(b_{j}-b_{k}\right)$. One can see from figure 2 that each pair of $(j, k)$ solitons propagate along the same characteristic directions determined by the group velocities $v_{j k}=\left(a_{j}-a_{k}\right) /\left(b_{j}-b_{k}\right)$, compare with fig. 1 .

\section{The RHP in multi-dimensions and Manakov rings}

Here we consider a RHP with canonical normalization whose sewing function $G(\vec{x}, t, \lambda)$ depends on several auxiliary parameters: $t$ and $\vec{x}=\left(x_{1}, \ldots, x_{n}\right)^{T}$.

$$
\begin{aligned}
\xi^{+}(\vec{x}, t, \lambda) & =\xi^{-}(\vec{x}, t, \lambda) G(\vec{x}, t, \lambda), \quad \lambda \in \mathbb{R} \\
\lim _{\lambda \rightarrow \infty} \xi^{ \pm}(\vec{x}, t, \lambda) & =\mathbb{1} .
\end{aligned}
$$


We intend to show, that by considering $n$-component vector $\vec{x}$ one can treat NLEE in $n+1$-dimensional space-time. For simplicity we fix up the dependence of $G(\vec{x}, t, \lambda)$ on $t$ and $\vec{x}$ by:

$$
i \frac{\partial G}{\partial x_{k}}=\lambda\left[J_{k}, G(\vec{x}, \lambda)\right] .
$$

The first example of Manakov ring was introduced in [29]. It is a special ring of commuting partial differential operators which was constructed using nonlocal RHP whose sewing function depended on several auxiliary variables and on two spectral variables. The ring we use here is more simple and easier to analyze.

Next we introduce

$$
\mathcal{J}_{k}^{ \pm}(\vec{x}, \lambda)=\xi^{ \pm}(\vec{x}, \lambda) J_{k} \hat{\xi}^{ \pm}(\vec{x}, \lambda) .
$$

Obviously $\mathcal{J}_{k}^{ \pm}(\vec{x}, \lambda)$ provide $r$ linearly independent analytic functions taking values in $\mathfrak{g}$ which commute between themselves:

$$
\left[\mathcal{J}_{k}^{+}(\vec{x}, \lambda), \mathcal{J}_{s}^{+}(\vec{x}, \lambda)\right]=\left[\mathcal{J}_{k}^{-}(\vec{x}, \lambda), \mathcal{J}_{s}^{-}(\vec{x}, \lambda)\right]=0
$$

for all $k, s=1, \ldots, r$.

Next we introduce the first order co-jets as elements of $\mathfrak{g}$ which are linear in $\lambda$ and are defined by:

$$
\begin{aligned}
\mathcal{J}_{k}^{(1)}(\vec{x}, \lambda) & =\left(\lambda \mathcal{J}_{k}(\vec{x}, \lambda)\right)_{+} \\
& =\lambda J_{k}+\left[J_{k}, Q(\vec{x})\right] .
\end{aligned}
$$

The subscript + here means that we take the asymptotic expansion of $\mathcal{J}_{k}(\vec{x}, \lambda)$ over the inverse powers of $\lambda$ and keep only the terms with non-negative powers.

Obviously to each of the co-jets we can relate a Lax operator $L_{k}$ :

$$
L_{k} \xi^{ \pm}(\vec{x}, \lambda) \equiv i \frac{\partial \xi^{ \pm}}{\partial x_{k}}-\lambda\left[J_{k}, \xi^{ \pm}(\vec{x}, \lambda)\right]+\left[J_{k}, Q(\vec{x})\right] \xi^{ \pm}(\vec{x}, \lambda)=0 .
$$

As a result the functions $\mathcal{J}_{k}^{ \pm}(\vec{x}, \lambda)$ satisfy:

$$
i \frac{\partial \mathcal{J}_{k}^{ \pm}}{\partial x_{k}}=\left[\mathcal{J}_{k}^{(1)}(\vec{x}, \lambda), \mathcal{J}_{k}^{ \pm}(\vec{x}, \lambda)\right] .
$$

The fact that all $L_{k}$ have the $\xi^{ \pm}(\vec{x}, \lambda)$ as fundamental analytic solutions means that they must commute identically with respect to $\lambda$, provided $Q(\vec{x})$ satisfies a certain set of PDE's. To check this fact and to derive those PDE's we calculate

$$
\frac{\partial}{\partial x_{k}}\left(\frac{\partial \xi^{ \pm}}{\partial x_{j}}\right)-\frac{\partial}{\partial x_{j}}\left(\frac{\partial \xi^{ \pm}}{\partial x_{k}}\right)
$$


and find that the set of PDE's satisfied by $Q(\vec{x})$ take the form:

$$
i\left[J_{k}, \frac{\partial Q}{\partial x_{j}}\right]-i\left[J_{j}, \frac{\partial Q}{\partial x_{k}}\right]+\left[\left[J_{j}, Q(\vec{x})\right],\left[J_{k}, Q(\vec{x})\right]\right]=0, \quad j=1, \ldots, r^{\prime} .
$$

for all $1 \leq k \neq j \leq r$. Thus for $r>3$ we get an overdetermined system of $r(r-1) / 2$ equations for the matrix-valued function $Q(\vec{x})$ of $r$ variables.

Let us assume that the number of auxiliary variables $r^{\prime}$ exceeds $r$ - the rank of the algebra $\mathfrak{g}$. In this case only $r$ of the Cartan generators $J_{k}$ can be linearly independent which means that at most $r$ of the auxiliary variables can be considered as independent; the rest will be linear combinations of them.

\section{1 $N$-wave resonant interaction in three space-time di- mensions}

This equations were discovered and analyzed at the end of the 1970's by Cornile and Kaup $[2,15]$. They are obtained as a particular case of our construction as follows. Choose $\mathfrak{g} \simeq \operatorname{su}(3)$ and fix up $\vec{x}=(t, x, y)^{T}$,

$$
\begin{array}{rlrl}
Q(\vec{x}) & =\left(\begin{array}{ccc}
0 & q_{12} & q_{13} \\
q_{21} & 0 & q_{32} \\
q_{31} & q_{32} & 0
\end{array}\right), & J_{1}=\operatorname{diag}\left(a_{1}, a_{2}, a_{3}\right), \\
J_{2}=\operatorname{diag}\left(b_{1}, b_{2}, b_{3}\right), & J_{3}=\operatorname{diag}\left(c_{1}, c_{2}, c_{3}\right),
\end{array}
$$

where $Q(\vec{x})=Q^{\dagger}(\vec{x})$ the constants $a_{j}, b_{j}, c_{j}$ satisfy $\operatorname{tr} J_{1}=\operatorname{tr} J_{2}=\operatorname{tr} J_{3}=0$ and

$$
a_{1}>a_{2}>a_{3}, \quad b_{1}>b_{2}>b_{3}, \quad c_{1}>c_{2}>c_{3},
$$

Then the PDE takes the form:

$$
\begin{array}{r}
2 \frac{\partial Q}{\partial t}-\left(\operatorname{ad}_{J_{2}}^{-1} \operatorname{ad}_{J_{1}}\right) \frac{\partial Q}{\partial x}-\left(\operatorname{ad}_{J_{3}}^{-1} \operatorname{ad}_{J_{1}}\right) \\
\frac{\partial Q}{\partial y}-i \operatorname{ad}_{J_{2}}^{-1}\left[\left[J_{1}, Q\right],\left[J_{2}, Q(\vec{x})\right]\right] \\
-i \operatorname{ad}_{J_{3}}^{-1}\left[\left[J_{1}, Q\right],\left[J_{3}, Q(\vec{x})\right]\right]=0
\end{array}
$$

where for any off-diagonal matrix $X$

$$
\left.\left.\operatorname{ad}_{J_{1}} X\right)_{j k} \equiv\left(\left[J_{1}, X\right]\right)_{j k}=\left(a_{j}-a_{k}\right) X_{j k}, \quad \operatorname{ad}_{J_{1}}^{-1} X\right)_{j k}=\frac{1}{a_{j}-a_{k}} X_{j k},
$$

and similarly for $J_{2}$ and $J_{3}$. In components eq. (5.11) reads

$$
\begin{aligned}
& 2 \frac{\partial q_{12}}{\partial t}-\frac{a_{1}-a_{2}}{b_{1}-b_{2}} \frac{\partial q_{12}}{\partial x}-\frac{a_{1}-a_{2}}{c_{1}-c_{2}} \frac{\partial q_{12}}{\partial y}+i \kappa_{12} q_{13} q_{23}^{*}=0, \\
& 2 \frac{\partial q_{13}}{\partial t}-\frac{a_{1}-a_{3}}{b_{1}-b_{3}} \frac{\partial q_{13}}{\partial x}-\frac{a_{1}-a_{3}}{c_{1}-c_{3}} \frac{\partial q_{13}}{\partial y}+i \kappa_{13} q_{12} q_{23}=0, \\
& 2 \frac{\partial q_{23}}{\partial t}-\frac{a_{2}-a_{3}}{b_{2}-b_{3}} \frac{\partial q_{23}}{\partial x}-\frac{a_{2}-a_{3}}{c_{2}-c_{3}} \frac{\partial q_{23}}{\partial y}+i \kappa_{23} q_{13} q_{12}^{*}=0,
\end{aligned}
$$


where

$$
\kappa_{j k}=-3\left(\frac{a_{k} b_{j}-a_{j} b_{k}}{b_{j}-b_{k}}+\frac{a_{k} c_{j}-a_{j} c_{k}}{c_{j}-c_{k}}\right) .
$$

The coefficient 2 multiplying the $t$-derivative can be removed by rescaling of $t$.

In the next example we have again $\mathfrak{g} \simeq \operatorname{sl}(3)$ with the notation

$$
Q(\vec{x})=i\left(\begin{array}{ccc}
0 & q_{3} & q_{2}^{*} \\
p_{3}^{*} & 0 & q_{1} \\
p_{2} & p_{1}^{*} & 0
\end{array}\right)
$$

and $J_{1}, J_{2}, J_{3}$ defined as before. But this time we impose a reduction $Q(\vec{x})=$ $-B Q^{\dagger}(\vec{x}) B$. In this case $B$ is a constant diagonal matrix with entries $B=$ $\operatorname{diag}\left(\sigma_{1}, \sigma_{2}, \sigma_{3}\right), \sigma_{i}= \pm 1$. This reduction gives $p_{1}=\sigma_{2} \sigma_{3} q_{1}, p_{2}=\sigma_{1} \sigma_{3} q_{2}$, $p_{3}=\sigma_{1} \sigma_{2} q_{3}$. It acts on the spectral parameter as $B(\lambda)=\lambda^{*}$ and on the eigenfunctions of the spectral problem as $B \psi^{\dagger}\left(\lambda^{*}\right) B=\hat{\psi}(\lambda)$.

The corresponding PDE (5.11) becomes

$$
\begin{array}{r}
2 \frac{\partial q_{3}}{\partial t}-\frac{a_{1}-a_{2}}{b_{1}-b_{2}} \frac{\partial q_{3}}{\partial x}-\frac{a_{1}-a_{2}}{c_{1}-c_{2}} \frac{\partial q_{3}}{\partial y}-\kappa_{12} \sigma_{2} \sigma_{3} q_{1}^{*} q_{2}^{*}=0 \\
2 \frac{\partial q_{2}}{\partial t}-\frac{a_{1}-a_{3}}{b_{1}-b_{3}} \frac{\partial q_{2}}{\partial x}+\frac{a_{1}-a_{3}}{c_{1}-c_{3}} \frac{\partial q_{2}}{\partial y}+\kappa_{13} q_{1}^{*} q_{3}^{*}=0 \\
2 \frac{\partial q_{1}}{\partial t}-\frac{a_{2}-a_{3}}{b_{2}-b_{3}} \frac{\partial q_{1}}{\partial x}+\frac{a_{2}-a_{3}}{c_{2}-c_{3}} \frac{\partial q_{1}}{\partial y}-\kappa_{13} \sigma_{1} \sigma_{2} q_{2}^{*} q_{3}^{*}=0
\end{array}
$$

This is the example studied by Kaup in [15]. We will present some explicit solutions of this system, using the dressing method for the underlying RHP. The one-soliton solution, corresponds to one pair of discrete eigenvalues, which, due to the chosen reduction are $\lambda_{1}^{+}=\mu_{1}+i \nu_{1}$ and $\lambda_{1}^{-}=\mu_{1}-i \nu_{1}$. The eigenfunction for the one-soliton solution is $[30,31,23]$

$$
\chi_{1}(\vec{x}, \lambda)=\left(1+\frac{\lambda_{1}^{-}-\lambda_{1}^{+}}{\lambda-\lambda_{1}^{-}} P\right) \chi_{0}(\vec{x}, \lambda), \quad \chi_{0}(\vec{x}, \lambda)=e^{-i \lambda_{1}^{+}\left(a_{p} t+b_{p} x+c_{p} y\right)},
$$

where

$$
P=\frac{|n\rangle\langle m|}{\langle m \mid n\rangle},
$$

with $|n\rangle=\chi_{0}\left(\vec{x}, \bar{\lambda}_{0}\right)\left|n_{0}\right\rangle$ where $\left|n_{0}\right\rangle$ is an arbitrary constant vector. The reduction on the eigenfunctions necessitates $|m\rangle=B\left|n^{*}\right\rangle$ and

$$
Q_{1 \mathrm{~s} ; j k}=-\left(1-\delta_{j k}\right) 2 i \nu_{1} P_{j k}
$$

In this case

$$
q_{1}(\vec{x})=2 \nu_{1} \sigma_{3} \frac{n_{2} n_{3}^{*}}{\Delta}, \quad q_{2}(\vec{x})=2 \nu_{1} \sigma_{3} \frac{n_{1}^{*} n_{3}}{\Delta}, \quad q_{3}(\vec{x})=2 \nu_{1} \sigma_{2} \frac{n_{1} n_{2}^{*}}{\Delta}
$$

where

$$
\begin{gathered}
n_{p}(\vec{x})=e^{-i \lambda_{1}^{+}\left(a_{p} t+b_{p} x+c_{p} y\right)} n_{0 p} \\
\Delta=\sigma_{1}\left|n_{1}\right|^{2}+\sigma_{2}\left|n_{2}\right|^{2}+\sigma_{3}\left|n_{3}\right|^{2},
\end{gathered}
$$


Here $n_{0 p}$ are three arbitrary constants. If we choose all $\sigma_{p}=1$ then $\Delta>0$ and all solutions are finite with decay at infinity. If $\sigma_{p}$ are of different signs then unstable (blow-up) solutions occur. Actually, in $1+1$ dimensions this equation describes plasma with blow-up instability, [23].

Further possible reduction is when the requirement of reality is imposed on the solutions. The simplest solution of this kind one can obtain by simply taking $\mu_{1}=0, \lambda_{1}^{+}=i \nu_{1}$, purely imaginary eigenvalue.

Similarly one can obtain other generalizations. For example, the generalization of (3.8) is the following 4 -wave equation:

$$
\begin{array}{r}
2 Q_{12 ; t}-\frac{a_{1}-a_{2}}{b_{1}-b_{2}} Q_{12 ; x}-\frac{a_{1}-a_{2}}{c_{1}-c_{2}} Q_{12 ; y}+i \kappa_{12} Q_{13} Q_{23}^{*}(t, x, y)=0 \\
2 Q_{13 ; t}-\frac{a_{1}}{b_{1}} Q_{13 ; x}-\frac{a_{1}}{c_{1}} Q_{13 ; y}-i \kappa_{13}\left(Q_{12} Q_{23}-Q_{14} Q_{23}^{*}(t, x, y)\right)=0 \\
2 Q_{14 ; t}-\frac{a_{1}+a_{2}}{b_{1}+b_{2}} Q_{14 ; x}-\frac{a_{1}+a_{2}}{c_{1}-c_{2}} Q_{14 ; y}-i \kappa_{14} Q_{13} Q_{23}(t, x, y)=0 \\
2 Q_{23 ; t}-\frac{a_{2}}{b_{2}} Q_{23 ; x}-\frac{a_{2}}{c_{2}} Q_{23 ; y}+i \kappa_{23}\left(Q_{14} Q_{13}^{*}+Q_{13} Q_{12}^{*}(t, x, y)\right)=0
\end{array}
$$

where

$$
\begin{array}{ll}
\kappa_{12}=\left(\frac{b_{2} a_{1}-a_{2} b_{1}}{b_{1}-b_{2}}+\frac{c_{2} a_{1}-a_{2} c_{1}}{c_{1}-c_{2}}\right), & \kappa_{13}=\left(\frac{b_{2} a_{1}-a_{2} b_{1}}{b_{1}}+\frac{c_{2} a_{1}-a_{2} c_{1}}{c_{1}}\right), \\
\kappa_{14}=\left(\frac{b_{2} a_{1}-a_{2} b_{1}}{b_{1}+b_{2}}+\frac{c_{2} a_{1}-a_{2} c_{1}}{c_{1}+c_{2}}\right), & \kappa_{23}=\left(\frac{b_{2} a_{1}-a_{2} b_{1}}{b_{2}}+\frac{c_{2} a_{1}-a_{2} c_{1}}{c_{2}}\right) .
\end{array}
$$

\section{Discussion and conclusions}

We have outlined the methods for construction of soliton solutions for the $N$ wave equations without and with additional reductions. The additional reductions may lead to new types of solitons which behave like the breathers of sine-Gordon. We have formulated our results for the 3 -wave and 4 -wave equations but there is no difficulty in extending these results to $N$-wave equations related to any simple Lie algebra.

Next we showed that these results can be easily extended for $N$-wave equations in higher dimensions. Obviously the soliton solutions that we have constructed will not be localized in multidimensions. The construction of localized solutions requires further studies.

\section{Acknowledgments}

It is our pleasure to thank Professor A. Constantin and Erwin Schrödinger Institute, Vienna, Austria where this paper was finished for the kind hospitality. The work of RI is supported by the Science Foundation Ireland (SFI), under Grant No. 09/RFP/MTH2144. We thank Professor A. V. Mikhailov for useful discussions. 


\section{References}

[1] A. M. Balk and E. V. Ferapontov, Invariants of 4-wave interactions, Physica D 65 (1993) 274-288.

[2] H. Cornile. Solutions of the Nonlinear Three-wave Equations in Three Dimensions. (preprint) (1978).

[3] E. V. Doktorov, S. B. Leble. Dressing method in Mathematical Physics. Mathematical Physics Studies Kontsevich, M., Porrati, M., Matveev, V.B., Sternheimer, D. (Eds.) vol 28. (2007), pp. 386. Springer, Berlin, Heidelberg, New York.

[4] E. V. Ferapontov, Isoparametric hypersurfaces in spheres, integrable nondiagonalizable systems of hydrodynamic type, and $N$-wave systems, Diff. Geom. Appl. 5 (1995) 335-369.

[5] A. S. Fokas and M. J. Ablowitz, "On the inverse scattering and direct linearization transform for the KP equation," Physics Letters 94A, (1983), $67-70$.

[6] Vladimir Gerdjikov, Georgi Grahovski, Nikolay Kostov. Second Order Reductions of $N$-wave interactions related to low-rank simple Lie algebras. Eds.: Ivailo M. Mladenov and Gregory L. Naber., Coral Press Scientific Publications, pp. 55-77, (2000).

[7] V. S. Gerdjikov, G. G. Grahovski, N. A. Kostov. Reductions of N-wave interactions related to low-rank simple Lie algebras. $I: \mathbb{Z}_{2}$-reductions. J. Phys. A: Math \& Gen. 34, 9425-9461 (2001).

[8] V. S. Gerdjikov, David J. Kaup. How many types of soliton solutions do we know? In the proceedings of Seventh Seventh International conference on Geometry, Integrability and Quantization, June 2-10, 2005, Varna, Bulgaria. Eds. Ivailo Mladenov, Manuel de Leon, Softex, Sofia (2006); pp. 11-34.

[9] V. S. Gerdjikov, D. J. Kaup, N. A. Kostov, T. I. Valchev. On classification of soliton solutions of multicomponent nonlinear evolution equations.

J. Phys. A: Math. Theor. 41 (2008) 315213 (36pp).

[10] V.S. Gerdjikov, N.A. Kostov, T.I. Valchev. N-Wave Equations with Orthogonal Algebras: $\mathbb{Z}_{2}$ and $\mathbb{Z}_{2} \times \mathbb{Z}_{2}$ Reductions and Soliton Solutions. SIGMA 3, paper 039 (2007); 19 pages. $\quad$ arXiv:nlin.SI/0703002.

[11] V. S. Gerdjikov, T. Valchev. Breather Solutions of $N$-wave Equations. Geometry, Integrability and Quantization, Eds: Ivailo Mladenov, Manuel de Leon. pp. 184-200, Softex, Sofia (2007). 
[12] V. S. Gerdjikov, G. Vilasi, A. B. Yanovski. Integrable Hamiltonian Hierarchies. Spectral and Geometric Methods Lecture Notes in Physics 748, Springer Verlag, Berlin, Heidelberg, New York (2008). ISBN: 978-3-54077054-1.

[13] E. Ibragimov, A.A. Struthers, D.J. Kaup, J.D. Khaydarov and K.D. Singer. Three-Wave Interaction Solitons in Optical Parametric Amplification Phys. Rev. E, 59, 6122-6137, 1999.

[14] Ivanov R., On the dressing method for the generalised Zakharov-Shabat system, Nuclear Phys. B 694 (2004), 509-524, math-ph/0402031.

[15] D.J. Kaup. A Method for Solving the Separable Initial Value Problem of the Full Three Dimensional Three-Wave Interaction, Stud. Appl. Math. 62, 75-83 (1980).

[16] D. J. Kaup, "A method for solving the separable initial value problem of the full threedimensional three-wave interaction," Stud. Appl. Math., 62, 75-83 (1980).

[17] D. J. Kaup, "The inverse scattering solution for the full three-dimensional three-wave resonant interaction," Physica 3D, i, No. i, 45-67 (1980).

[18] S. V. Manakov, "The inverse scattering transform for the time-dependent Schrodinger equation and Kadomtsev-Petviashvili equation," Physica 3D, 2, 420-427 (1981).

[19] S. V. Manakov, P. Santini, and L. A. Takhtajan, "Long-time behavior of the solutions of the Kadomtsev-Petviashvili equation (two-dimensional KdV equation)," Phys. Lett., 74A, 451-454 (1980).

[20] S. V. Manakov and V. E. Zakharov, "Soliton theory," in: Physics Review, Vol. i, I. M. Khalatnikov (ed.), London (1979), pp. 133-190;

S. V. Manakov and V. E. Zakharov, Exact theory of resonant interaction of wave packets in nonlinear media. Preprint of Novosibirsk Institute of Nuclear Physics 74-41 (1974) (unpublished).

[21] A. V. Mikhailov and V. E. Zakharov, "On the integrability of classical spinor models in two-dimensional space-time," Commun. Math. Phys., 744, 21-40 (1980).

[22] A. Nachman and M. Ablowitz, Multidimensional Inverse scattering for First-Order Systems, Stud. Appl. Math. 71 (1984) 251-262.

[23] Novikov, S. P., Manakov, S. V., Pitaevsky, L. P. and Zakharov, V. E. Theory of solitons: the inverse scattering method. New York, Plenum, 1984

[24] P.J. Vassiliou, Method for solving the multidimensional $n$-wave resonant equations and geometry of generalised Darboux-Manakov-Zakharov systems, Stud. Appl. Math. 126 (2010) 203-243. 
[25] V. E. Zakharov, "Exact solutions of the problem of parametric interaction of wave packets," Dokl. Akad. Nauk SSSR, 228, No. 6, 1314-1316 (1976).

[26] V. E. Zakharov, "The inverse scattering method," in: Solitons, R. K. Bullough and P. J. Caudrey (editors), Springer-Verlag, Berlin (1980), pp. 243286.

[27] V. E. Zakharov, "Integrable systems in multidimensional spaces," in: Mathematical Problems in Theoretical Physics, Lecture Notes in Phys., Vol. 153, Springer-Verlag, Berlin (1982), pp. 190-216.

[28] Zakharov V E., Manakov S V. Zh. Exp. Teor. Fiz. 69, 1654-1673 (1975); Manakov S V., Zakharov V E. Zh. Exp. Teor. Fiz. 71, 203-215 (1976).

[29] V. E. Zakharov and S. V. Manakov. Multidimensional nonlinear integrable systems and methods for constructing their solutions. Zapiski Nauchnykh Seminarov Leningradskogo Otdeleniya Matematicheskogo Instituta im. V. A. Steklova AN SSSR, Vol. 133, pp. 77-91, 1984.

[30] V. E. Zakharov and A. B. Shabat, "A scheme for integrating nonlinear evolution equations of mathematical physics by the inverse scattering method. I," Funkts. Anal. Prilozhen., 8, No. 3, 43-53 (1974).

[31] V. E. Zakharov and A. B. Shabat, "Integration of the nonlinear equations of mathematical physics by the inverse scattering method," Funkts. Anal. Prilozhen., 13, No. 3, 13-22 (1979). 